\title{
MEPE/OF45 as a new target for sensitizing human tumour cells to DNA damage inducers
}

\author{
P Zhang ${ }^{1,5}, \mathrm{H}$ Wang ${ }^{2}$, PSN Rowe ${ }^{3}$, B Hu ${ }^{4}$ and Y Wang ${ }^{*, 2}$ \\ 'Department of Radiation Oncology, Thomas Jefferson University, Philadelphia, PA 19107, USA; ²Department of Radiation Oncology of Winship Cancer Institute, \\ Emory University School of Medicine, Atlanta, GA 30322, USA; ${ }^{3}$ Department of Intemal Medicine, Kidney Institute and Division of Nephrology, University of Kansas \\ Medical Center, Kansas City, KS 66 I60, USA; ${ }^{4}$ Department of Medical Molecular Biology, Beijing Institute of Biotechnology, Beijing 100850, China
}

BACKGROUND: We recently identified matrix extracellular phosphoglycoprotein/osteoblast factor 45 (MEPE/OF45) as a new cofactor of CHKI in rat cells. The aim of this study was to determine the role of human MEPE/OF45 (hMEPE/OF45 has 50\% homology with rat MEPE/OF45 (rMEPE/OF45)) in affecting the sensitivity of human tumour cells to DNA damage.

METHODS: hMEPE/OF45 expression in different human tumour cell lines and its relevance to the resistance of cell lines to DNA damage inducers such as ionising radiation (IR) or camptothecin (CPT) were assessed. Cells lines stably expressing wild-type MEPE/OF45 or mutant MEPE/OF45 (with the CHKI interactive key domain (amino acids 488-507) deleted) were established. Cell survival, $\mathrm{G}_{2}$ accumulation, $\mathrm{CHKI}$ half-life and the $\mathrm{CHKI}$ level in ligase 3 complexes were examined.

RESULTS: hMEPE/OF45 expression correlates with the resistance of cell lines to IR or CPT. Upregulating wild-type hMEPE/OF45 (but not mutant hMEPE/OF45) could stabilize CHKI by reducing $\mathrm{CHKI}$ interaction for its E3 ligases Cull or Cula4A; it increases the $\mathrm{G}_{2}$ checkpoint response and increases the resistance of tumour cells to IR or CPT treatment.

CONCLUSION: hMEPE/OF45 could be a new target for sensitizing tumour cells to radiotherapy or chemotherapy.

British Journal of Cancer (2010) I 02, 862-866. doi:I0.I038/sj.bjc.6605572 www.bjcancer.com

Published online 9 February 2010

(c) 2010 Cancer Research UK

Keywords: MEPE/OF45; CHKI; DNA damage

Matrix extracellular phosphoglycoprotein/osteoblast factor 45 (MEPE/OF45) was originally cloned from a human oncogenetic hypophosphataemia tumour (Rowe et al, 2000) and then identified in rat bone-marrow-derived osteoblasts (Petersen et al, 2000). The murine homologue of MEPE/OF45 was reported the following year (Argiro et al, 2001). Since the identification of MEPE/OF45, its function related to bone metabolism has been widely investigated. We previously identified that MEPE/OF45, as a cofactor of CHK1, protects cells from DNA damage-induced killing in rat embryo fibroblast cells (Liu et al, 2009). However, because human MEPE/ OF45 (hMEPE/OF45) has only 50\% homology with rat MEPE/ OF45 (rMEPE/OF45), it remained unclear whether hMEPE/OF45 has similar function to rMEPE/OF45.

CHK1 is one of the essential checkpoint proteins involved in cellular response to multiple DNA damage inducers (Liu et al, 2000; Zhao et al, 2002). It is believed that upregulated CHK1 protects cells from DNA damage-induced cell killing, including that induced by ionising radiation (IR) and camptothecin (CPT) (Hu et al, 2001, 2005a; Zhao et al, 2002; Wang et al, 2002b; Brown

* Correspondence: Dr Y Wang, Department of Radiation Oncology, Winship Cancer Institute of Emory University School of Medicine, 1365 Clifton Road, NE, Atlanta, GA 30322, USA;

E-mail: yawang@radonc.emory.org

${ }^{5}$ Current address: Department of Laboratory Medicine, Mayo Clinic College of Medicine, Rochester, MN, USA.

Received 29 October 2009; revised 15 January 2010; accepted 18 January 2010; published online 9 February 2010 and Baltimore, 2003), by promoting homologous recombination repair (HRR) (Sorensen et al, 2005; Hu et al, 2005b). Despite the importance of $\mathrm{CHK} 1$ in DNA damage response, the regulation of CHK1 in mammalian cells is not well understood, partly because of its essential nature (Liu et al, 2000; Lam et al, 2004).

In this study, on the basis of our previous report about the new role of rMEPE/OF45 in protecting cells from DNA damage-induced killing (Liu et al, 2009), we investigate whether hMEPE/OF45 is generally expressed in different types of tumour cells, whether the expression levels were relevant to the resistance of tumour cells to DNA damage inducers and whether, similar to rMEPE/OF45, hMEPE/OF45 (as a cofactor of CHK1) could be a new target for sensitizing human tumour cells to DNA damage inducers.

\section{MATERIALS AND METHODS}

\section{Cell lines and antibodies}

All human tumour cell lines used in this study were derived from ATCC. These cell lines were grown in DMEM medium supplemented with $10 \%$ calf serum. The antibody against hMEPE/OF45 was as described (Rowe et al, 2000, 2004). Purified HA-tagged JNK2 protein (sc-4062) and antibodies against CHK1 (sc-8408 and sc-7898), HA (sc-805, sc-7392), PCNA (sc-56), $\alpha$-Tubulin (sc-8035), Cul4 (sc-10782) and ubquitin (Ub) (sc-9133) were purchased from Santa Cruz Biotechnology Inc (Santa Cruz, CA, USA). The antibody against Cull was purchased from Zymed Laboratories (San Francisco, CA, USA) and the antibody against 
H2A (07-146) was purchased from Upstate Biotechnology (Lake Placid, NJ, USA).

\section{Plasmid construction and transfection}

The full-length cDNA of wild-type $h M E P E / O F 45$ or mutant hMEPE/OF45 (with the sequence encoding 488-507 amino acids (aa) deleted) was ligated with HA tag at the $\mathrm{C}$ terminus by PCR. $h M E P E / O F 45$ was then cloned into pLenti6/V5-D-TOPO vector (Invitrogen), according to the manufacturer's protocol. The HA tag was inserted into the plasmid. The primers used for wild-type hMEPE/OF45 are: forward, $5^{\prime}$-CACCATGGGAGTTTTCTGTGTGG GACTACTCCTTTTCAGTGTGACCTG-3' and reverse, 5'-AGGTCC TCCCAGGCTGGCATAGTCAGGCACGTCATAAGGATAGTCACCA TCGCTCTCACTTGAAC- $3^{\prime}$. The procedure to construct mutant hMEPE/OF45 has been described in Supplementary Figure S1. The primers for fragment 1 (1-477 aa) are F1, ACGGATCCATGCGA GTTTTCTGTGTGGGACTACTCC and R1, TACTGTCATCCTTATT CCGTGTAGA, and for fragment 2 (507-514 aa) are F2, ACGGAAT AAGGATGACAGTAGTGAGTCATC and R2, AAGAATTCGTCACC ATCGCTCTCACTTG. For lentivirus production, pLenti6/V5-DTOPO was co-transfected with packaging vectors into human 293 cells and the supernatant was harvested after $48 \mathrm{~h}$. The virus was filtered through a Millex-HV PVDF filter (hole diameter: $0.45 \mu \mathrm{M}$ ). Titres were determined by infecting 293 cells with a serial dilution of the concentrated virus. For a typical preparation, the titre was approximately 2 - to $7 \times 10^{4} \mathrm{TU} \mathrm{ml}^{-1}$. For infection of 293 cells, $30-50 \%$ confluent cells were incubated with a suitable amount of virus particles and $10 \mu \mathrm{g} \mathrm{ml}^{-1}$ polybrene for $8-16 \mathrm{~h}$ at $37^{\circ} \mathrm{C}$. A volume of $10 \mu \mathrm{g} \mathrm{ml}^{-1}$ of blasticidin (Invitrogen) was used to select stably expressed cells.

\section{Immunoprecipitation}

Immunoprecipitation was performed as described previously (Wang et al, 2002a). The samples were washed with buffer $\left(0.5 \% \mathrm{NP}-40,1 \mathrm{~mm} \mathrm{Na} \mathrm{VO}_{4}, 5 \mathrm{mM} \mathrm{NaF}\right.$ and $0.2 \mathrm{mM}$ PMSF in PBS buffer) and boiled in $30 \mu \mathrm{l}$ of protein loading buffer. They were loaded onto $10 \%$ Tris-PAGE gel, followed by the standard western blot.

\section{Cell sensitivity to DNA damage inducers and flow} cytometry measurement

Cell sensitivity to IR or to CPT was determined by clonogenic assay as described previously (Hu et al, 2001; Wang et al, 2002b). The $\mathrm{G}_{2}$ accumulation of cells after radiation was detected by flow cytometry as described previously ( $\mathrm{Hu}$ et al, 2001). Briefly, $2 \times 10^{5}$ cells were plated in $60 \mathrm{~mm}$ dishes, with $3 \mathrm{ml}$ of growth medium. After $30 \mathrm{~h}$, the cells were exposed to $6 \mathrm{~Gy}$ and returned to $37^{\circ} \mathrm{C}$. At different times thereafter, the cells were trypsinised and fixed in $70 \%$ ethanol. Cells were stained in solution $\left(62 \mu \mathrm{g} \mathrm{ml}^{-1} \mathrm{RNase} \mathrm{A}\right.$, $40 \mu \mathrm{g} \mathrm{ml}^{-1}$ propidium iodide and $0.1 \%$ Triton X-100 in PBS buffer) at room temperature for $1 \mathrm{~h}$. The distribution of cells in the cell cycle was measured using a flow cytometer (Coulter Epics Elite, Beckman Coulter Inc., Atlanta, GA, USA).

\section{Statistical analysis}

Differences between treatment groups were analysed using statistical software ( $t$-test). $P$-values $<0.05$ were regarded as significant.

\section{RESULTS AND DISCUSSION}

MEPE/OF45 presents in all kinds of human dividable cell lines tested in our laboratory

We previously identified that rMEPE/OF45 could protect rat cells from DNA damage-induced killing (Liu et al, 2009). However, because the amino-acid sequence of hMEPE/OF45 (525 amino acid) has $\sim 50 \%$ homology compared with that of rMEPE/OF45 (435 amino acid) (Argiro et al, 2001), it remained unclear whether hMEPE/OF45 has a function similar to rMEPE/OF45. To investigate whether MEPE/OF45 was important for human cells to respond to DNA damage, we examined the MEPE/OF45 expression level in different human cell lines, including primary fibroblast cells, transformed fibroblast cells, transformed lymphoblast cells and tumour cell lines from different human tissues. These cell lines all showed a positive expression of MEPE/OF45, although the levels of expression varied (Figure 1A), indicating that MEPE/OF45 expresses in replicating cells. The signals of human MEPE/OF45 showed two bands (Figure 1A), which might be due to alternatively
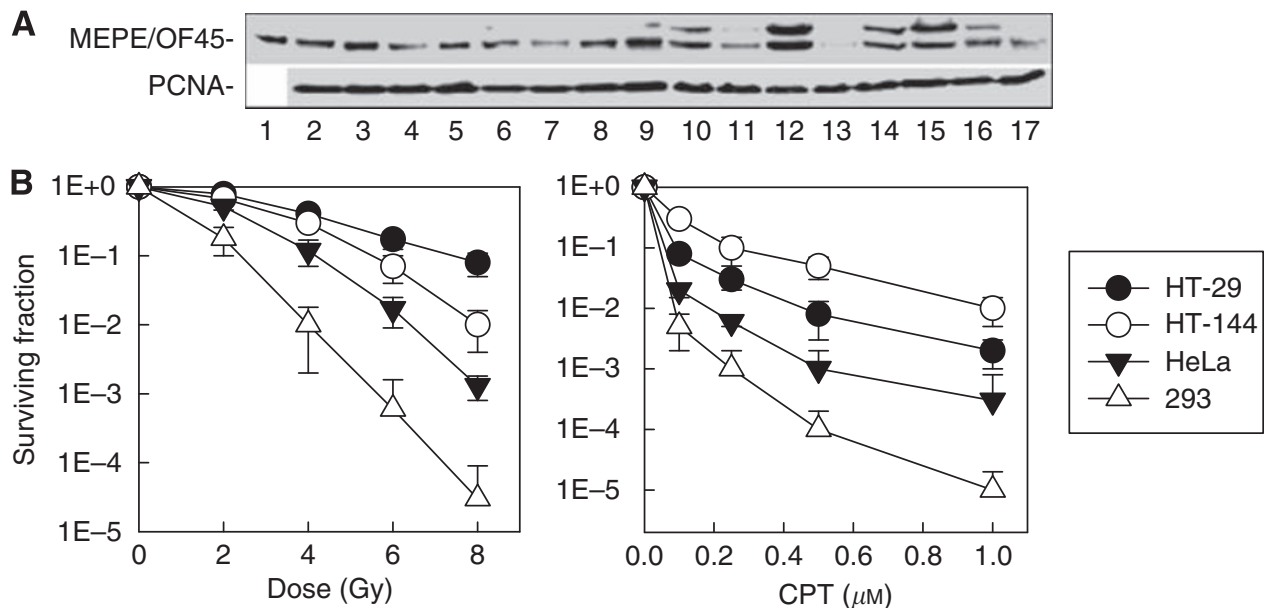

Figure I MEPE/OF45 expressed in dividable human cells. (A) Whole-cell lyses were prepared. The antibody against hMEPE/OF45 was as described (Rowe et al, 2000, 2004). Data shown are the images of western blot analysis. Expression of MEPE/OF45 in human normal and tumour cell lines. I, purified MEPE/OF45 protein (Rowe et al, 2000); 2. MRC5 (primary human fibroblast); 3. MRC5-SVI (transformed MRC5); 4, C3-ABR (transformed human lymphoblasts); 5, HeLa (human cervical cancer); 6, HCCI937 (breast cancer); 7, U87MG glioblastoma; 8, SAOS2 (osteosarcoma); 9, HT-29 (colorectal cancer); I , A549 (lung carcinoma); I I, PC-3 (prostate cancer); I2, HT- I44 (melanoma); I 3, 293 (kidney cancer); I4, SQ20B (laryngeal cancer); I 5, Capan- I (pancreatic cancer); 16, OVCARIO (ovarian adenocarcinoma); I7, MCF-7 (breast cancer). (B) Sensitivities of different human cell lines to DNA damage inducers. The sensitivities of cell lines to various doses of IR or CPT exposure $(24 \mathrm{~h})$ were examined using clonogenic assay. Bars show the mean \pm s.e. of the percentage of colonies from non-treated control cells. Data were obtained from three independent experiments. 
spliced $M E P E / O F 45 s$. Our group recently identified a new exon of $h M E P E / O F 45$ between formerly identified exons 3 and 4 (GenBank accession no. DQ854717). The functions of the different forms of MEPE/OF45 need to be investigated further. Interestingly, the levels of MEPE/OF45 in different human tumour cell lines broadly correlate with the resistance of cell lines to DNA damage inducers (Figure 1B). The higher the level of MEPE/OF45 was in the cell line, the more resistant the cell line was to IR or CPT. There were 293 cells with the lowest level of MEPE/OF45, and they were most sensitive to IR or CPT. In addition, we reported that knocking down MEPE/OF45 could sensitise HeLa cells to DNA damage inducers
(Liu et al, 2009). These results indicate that MEPE/OF45 protects human cells from DNA damage-induced killing.

\section{Human MEPE/OF45 interacts with CHK1 and stabilises CHK1}

We previously reported that rMEPE/OF45 protected cells from DNA damage-induced killing through its interaction with CHK1 (Liu et al, 2009). We wanted to determine whether similar to rMEPE/OF45, hMEPE/OF45 could also interact with CHK1. To study the relationship of hMEPE/OF45 with CHK1, we first
A

A $\mathrm{CHK} 1$

hMEPE/OF45-

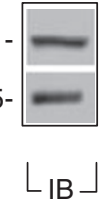

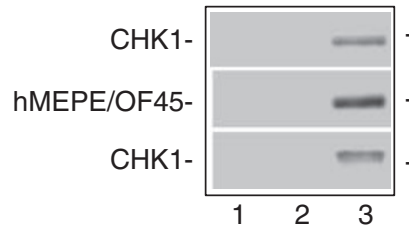

-hMEPE/OF45 IP

$-\mathrm{CHK} 1 \mathrm{IP}$

- CHK1 IP

B
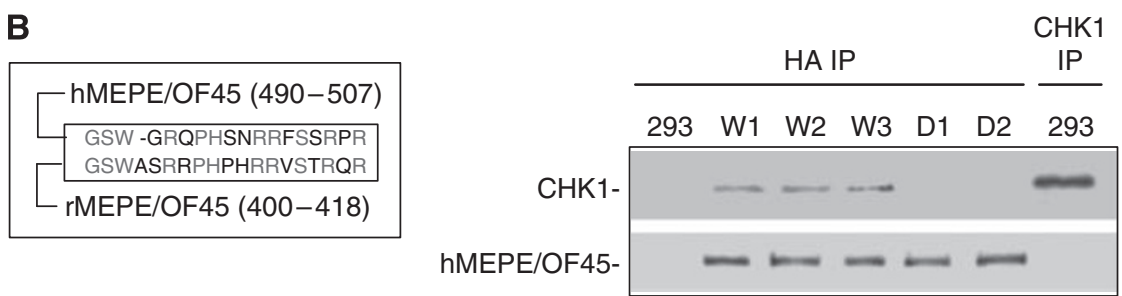

C
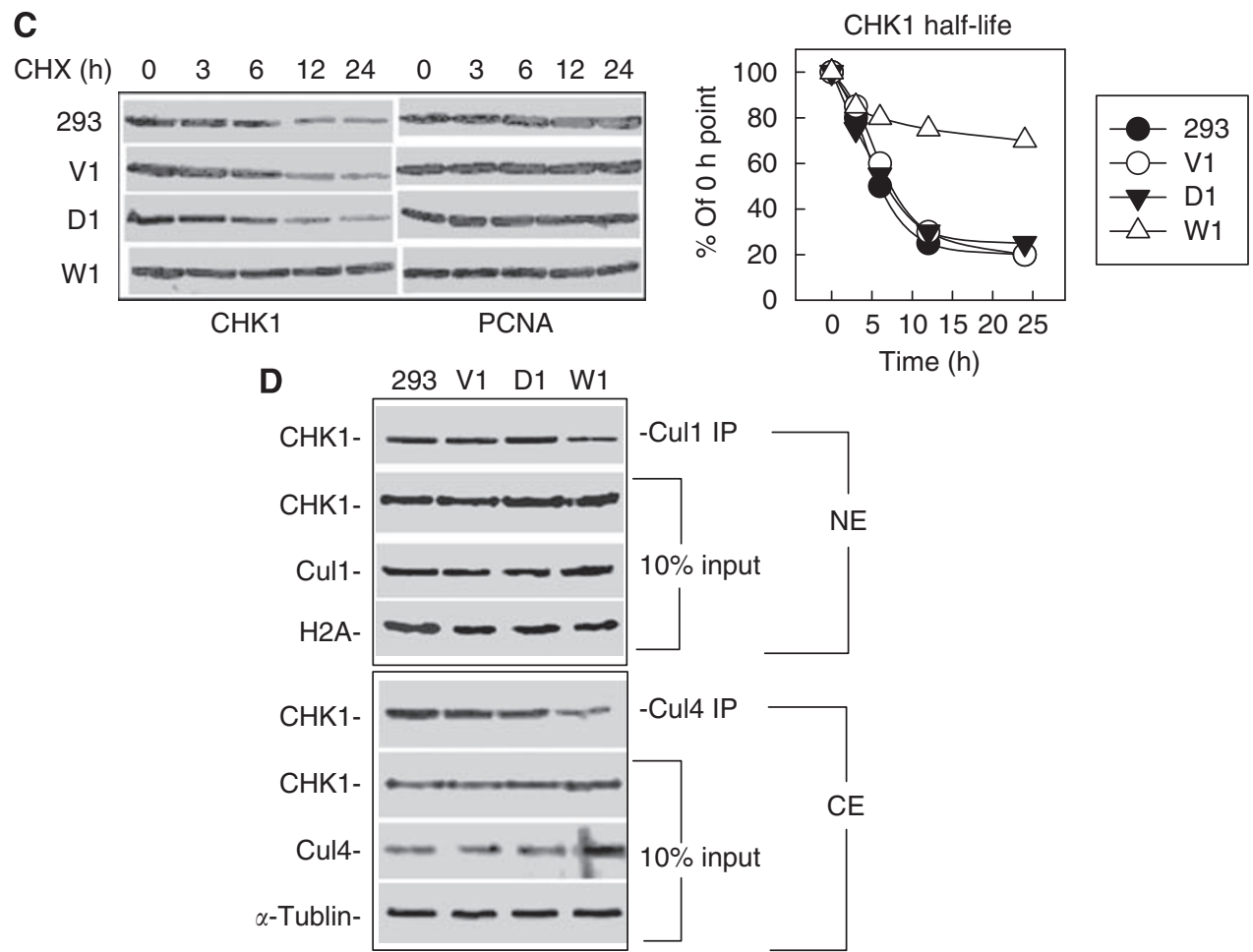

Figure 2 MEPE/OF45 interacts with CHKI and stabilises CHKI. (A) Whole-cell lyses were prepared from HeLa cells. Signals were detected from immunoprecipitation (IP) results. Lanes $\mathrm{I}$ and 2 show the results from IP experiments that used general mouse or rabbit serum as the negative controls. Lane 3 indicates the western blot signals of CHKI or of MEPE/OF45 from MEPE/OF45 or CHKI antibody-immunoprecipitated complex. (B) Left: the corresponding amino-acid sequence of rMEPE/OF45 (400-4l8) in hMEPE/OF45 is 490-507. Right: MEPE/OF45 interacts with CHKI in human 293 cell lines. Western blot (IB) with CHKI or with MEPE/OF45 antibody was used to detect the signals of MEPE/OF45 interacting with CHKI from CHKI or HA immunoprecipitated (IP) samples. VI: 293 cells transfected with vector alone; WI -W3: 293 cells transfected with wild-type hMEPE/OF45; DI -D2: 293 cells transfected with mutant hMEPE/OF45 (amino acid 490-507) deleted. (C) CHKI half-life in different cell lines: cells were treated with $100 \mu \mathrm{g} \mathrm{ml}^{-1} \mathrm{CHX}$ at different times. Whole-cell lyses were prepared. CHKI signals were detected by western blot. The CHKI levels were plotted using Phosphorlmager (GE Healthcare Bioscience Corp., Piscataway, NJ, USA) with software (ImageQuant). (D) MEPE/OF45 affects CHKI interacting with E3 ligases in human cells. Extracts were prepared from these cells using an NE-PER kit (Pierce, Rockford, IL, USA). The extracts (500 $\mu \mathrm{g}$ of cytoplasmic extracts or $300 \mu \mathrm{g}$ of nucleic extracts) were mixed with protein A that was conjugated with Cull or Cul4 antibody for IP. CHKI antibody was used for immunoblotting. $\mathrm{H} 2 \mathrm{~A}$ or $\alpha$-tubulin was used as an indicator for nuclear or cytoplasmic extracts. 
synchronised HeLa cells and examined the expression of MEPE/ OF45 at different phases of the cell cycle, as CHK1 expression depends on the cell cycle (Kaneko et al, 1999). The results showed that similar to CHK1, MEPE/OF45 expressed low in the $\mathrm{G}_{1}$ phase and high in the $S$ and $G_{2}$ phases (Supplementary Figure S2), suggesting a correlation between these two proteins. We further examined the interaction between hMEPE/OF45 and CHK1 using an immunoprecipitation (IP) approach in HeLa cells. The results showed that, similar to rMEPE/OF45, hMEPE/OF45 also interacted with CHK1 (Figure 2A). The key domain of rMEPE/OF45 was $400-418$ amino acids (Liu et al, 2009). The corresponding aminoacid sequence in hMEPE/OF45 is 490-507 (Argiro et al, 2001; Figure $2 \mathrm{~B}$, left). To study whether CHK1 was stabilised when interacting with hMEPE/OF45, as it was when interacting with rMEPE/ OF45, we generated the constructs encoding HA-tagged either wild-type hMEPE/OF45 or mutant hMEPE/OF45 with amino acid 488-507 deleted (Supplementary Figure S1). We expressed the wild-type hMEPE/OF45 or mutant hMEPE/OF45 in 293 cells, as this cell line had the lowest level of MEPE/OF45 in the human tumour cell lines tested in our laboratory (Figure 1A). The results showed that wild-type hMEPE/OF45 but not mutant hMEPE/OF45 could interact with $\mathrm{CHK} 1$ (Figure 2B, right), indicating that the 488-507 amino acids are the key domain for hMEPE/OF45 to interact with CHK1.

We were then interested in determining whether similar to the key domain of rMEPE/OF45, the key domain of hMEPE/OF45 also had an essential role in stabilising CHK1 by reducing the interaction of CHK1 for its E3 ligases, Cul1 or Cul4A. For this, we first compared the half-life of CHK1 between wild-type hMEPE/OF45-expressing cells and mutant hMEPE/OF45-expressing cells.

The results showed that the half-life of CHK1 in the wild-type hMEPE/OF45-expressing cells (W1) is longer than that in parental cells (293), the 293 cells transfected with the vector alone (V1) and the 293 cells transfected with mutant hMEPE/OF45 (D1) (Figure $2 \mathrm{C}$ ). These results indicate that the interaction domain is essential for hMEPE/OF45 to stabilise CHK1. We then compared the CHK1 levels in its E3 ligase complexes with wild-type hMEPE/ OF45-expressing cells and mutant hMEPE/OF45-expressing cells. Results showed that although the total levels of CHK1 in either cytoplasmic extracts (CE) or nuclear extracts (NE) are similar to all types of cells $(293, \mathrm{~V} 1, \mathrm{D} 1$ and W1), the CHK1 levels in Cul1 or Cul4A IP complexes in W1 cells were less than that in 293, V1 or D1 cells (Figure 2D). Additional data showed that when hMEPE/ OF45 was knocked down, ubiquitinated CHK1 was increased (Supplementary Figure S3A and $\mathrm{B}$ ). These results indicate that although hMEPE/OF45 has $\sim 50 \%$ homology with rMEPE/OF45, its $\mathrm{C}$-terminal amino-acid sequence, including the key domain to interact with $\mathrm{CHK} 1$, is relatively conserved, and similarly functions to stabilise CHK1.

\section{Wild-type but not mutant hMEPE/OF45 renders tumour cells resistant to DNA damage inducers}

To further examine whether hMEPE/OF45 was similar to rMEPE/ OF45, and could protect cells from DNA damage-induced killing through interaction with CHK1, we compared the effects of wildtype hMEPE/OF45 and mutant hMEPE/OF45 on the $\mathrm{G}_{2}$ checkpoint response and the sensitivity of 293 cells to DNA damage inducers. The results showed that wild-type hMEPE/OF45 could increase the $\mathrm{G}_{2} / \mathrm{M}$ accumulation of cells after IR, but not mutant hMEPE/OF45 (Figure 3A). Similarly, knocking down hMEPE/OF45 reduced IR-induced $\mathrm{G}_{2}$ accumulation (Supplementary Figure S3A and C). These results further support that hMEPE/OF45 functions through the CHK1 pathway to affect cell response to DNA damage, because this kind of checkpoint response, $\mathrm{G}_{2}$-phase cell accumulation, is ATM independent (Xu et al, 2002) but CHK1 dependent (Wang et al, 2003). More importantly, the results showed that wild-type hMEPE/OF45 could increase resistance of the cells to either IR or CPT treatment, but not mutant hMEPE/OF45 (Figure 3B). The $P$-values between 293W (including 1 and 2) and other groups are $<0.01$. These results indicate that hMEPE/OF45 protects cells from

A Time (h)
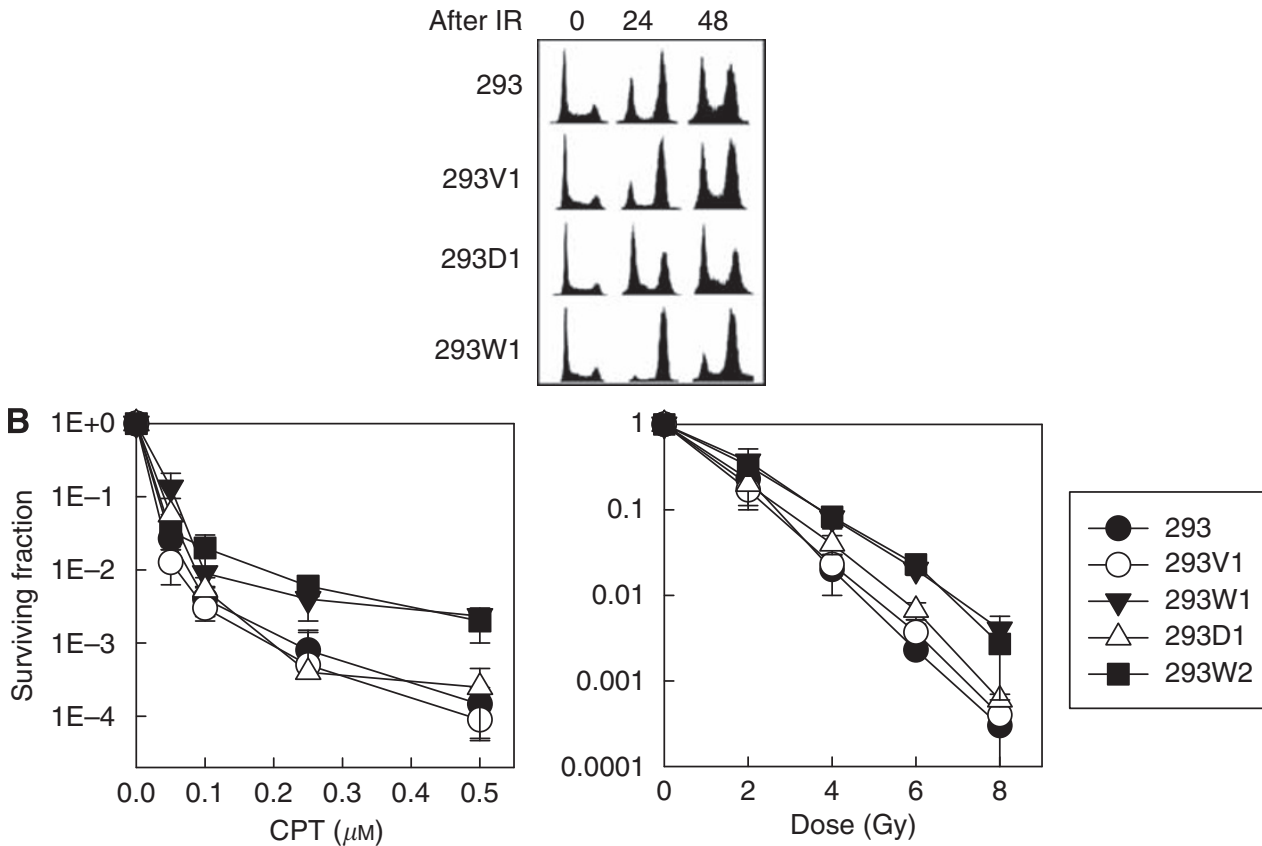

Figure 3 Upregulating wild-type MEPE/OF45 but not mutant MEPE/OF45 deleted the key domain that interacted with CHKI, making the tumour cells resistant to DNA damage inducers. (A) Comparison of the $\mathrm{G}_{2}$ accumulation of 293 cell lines $(293,293 \mathrm{VI}, 293 \mathrm{WI}$ and $293 \mathrm{DI}$ ) at different times after IR (6 Gy). The P-values between 293W (including I and 2) and other groups are <0.0 I. (B) Comparison of the sensitivities of 293, 293VI, 293W I, 293D I and 293W2 cells to IR or CPT treatment (I6 h). Data represented the average of three independent experiments and were expressed as the percentage of cell colonies without treatment. Data were obtained from three independent experiments. 
DNA damage-induced killing depending on its interaction with CHK1. It is known that CHK1 promotes HRR through regulation of checkpoint activation (Sorensen et al, 2005; Hu et al, 2005b). We believe that hMEPE/OF45 increases the CHK1 level by stabilising CHK1, which promotes HRR and protects cells from DNA damageinduced killing.

\section{CONCLUSION}

Taken together, the results of our study indicate that MEPE/OF45 protecting human cells from IR- or CPT-induced killing mainly depends on its interaction with CHK1. The specific molecular mechanism for this protective role of MEPE/OF45 could be linked to MEPE/OF45 stabilising $\mathrm{CHK} 1$ by reducing $\mathrm{CHK} 1$ degradation. Because CHK1 is an essential gene (Liu et al, 2000; Lam et al, 2004) and MEPE/OF45 is not (Gowen et al, 2003), our study results indicate that MEPE/OF45 is a new cofactor of CHK1, which could be a new target with less side effects for sensitizing

\section{REFERENCES}

Argiro L, Desbarats M, Glorieux FH, Ecarot B (2001) MEPE, the gene encoding a tumor-secreted protein in oncogenic hypophosphatemic osteomalacia, is expressed in bone. Genomics 74: $342-351$

Brown EJ, Baltimore D (2003) Essential and dispensable roles of ATR in cell cycle arrest and genome maintenance. Genes Dev 17: 615-628

Gowen LC, Petersen DN, Mansolf AL, Qi H, Stock JL, Tkalcevic GT, Simmons HA, Crawford DT, Chidsey-Frink KL, Ke HZ, McNeish JD, Brown TA (2003) Targeted disruption of the osteoblast/osteocyte factor 45 gene (OF45) results in increased bone formation and bone mass. J Biol Chem 278: $1998-2007$

Hu B, Han S-Y, Wang X, Ottey M, Potoczek MB, Dicker A, Huebner K, Wang $Y$ (2005a) Involvement of the Fhit gene in the ionizing radiationactivated ATR/CHK1 pathway. J Cell Physiol 202: 518-523

Hu B, Wang H, Wang X, Lu H-R, Huang C, Powell SN, Huebner K, Wang Y (2005b) Fhit and CHK1 have opposing effects on homologous recombination repair. Cancer Res 65: 8613-8616

Hu B, Zhou XY, Wang X, Zeng ZC, Iliakis G, Wang Y (2001) The radioresistance to killing of A1-5 cells derives from activation of the Chk1 pathway. J Biol Chem 276: $17693-17698$

Kaneko Y, Watanabe N, Morisaki H, Akita H, Fujimoto A, Tominaga K, Tachibana K, Ikeda K, Nakanishi M (1999) Cell cycle-dependent and ATMindependent expression of human Chk1 kinase. Oncogene 18: 3673-3681

Lam MH, Liu Q, Elledge SJ, Rosen JM (2004) Chk1 is haploinsufficient for multiple functions critical to tumor suppression. Cancer Cell 6: 45-59

Liu Q, Guntuku S, Cui XS, Matsuoka S, Cortez D, Tamai K, Luo G, Carattini-Rivera S, DeMayo F, Bradley A, Donehower LA, Elledge SJ (2000) Chk1 is an essential kinase that is regulated by Atr and required for the $\mathrm{G}_{2} / \mathrm{M}$ DNA damage checkpoint. Genes Dev 14: $1448-1459$

Liu S, Wang H, Wang X, Lu L, Gao N, Rowe P, Hu B, Wang Y (2009) MEPE/ OF45 protects cells from DNA damage induced killing via stabilizing CHK1. Nucleic Acids Res 37: 7447-7454 tumour cells to DNA damage inducers and could benefit cancer treatment.

\section{ACKNOWLEDGEMENTS}

We thank Ms Doreen Theune for editing this paper. This work was supported by grants from the National Basic Research Program of China (2005CB522506 and 2007CB914604 to BH), the National Natural Science Foundation of China (30770651, 30670616 and 30370441 to $\mathrm{BH}$ ) and a grant from the National Institutes of Health of USA (GM080771 to YW).

\section{Conflict of interest}

The authors declare no conflict of interest.

Supplementary Information accompanies the paper on British Journal of Cancer website (http://www.nature.com/bjc)
Petersen DN, Tkalcevic GT, Mansolf AL, Rivera-Gonzalez R, Brown TA (2000) Identification of osteoblast/osteocyte factor 45 (OF45), a bone-specific cDNA encoding an RGD-containing protein that is highly expressed in osteoblasts and osteocytes. J Biol Chem 275: $36172-36180$

Rowe PSN, de Zoysa PA, Dong R, Wang HR, White KE, Econs MJ, Oudet CL (2000) $M E P E$, a new gene expressed in bone marrow and tumors causing osteomalacia. Genomics 67: 54-68

Rowe PSN, Kumagai Y, Gutierrez G, Garrett IR, Blacher R, Rosen D, Cundy J, Navvab S, Chen D, Drezner MK (2004) MEPE has the properties of an osteoblastic phosphatonin and minhibin. Bone 34: 303-319

Sorensen CS, Hansen LT, Dziegielewski J, Syljuasen RG, Lundin C Bartek J, Helleday T (2005) The cell-cycle checkpoint kinase Chk1 is required for mammalian homologous recombination repair. Nat Cell Biol 7: $195-201$

Wang H, Wang X, Zhou XY, Chen DJ, Li GC, Iliakis G, Wang Y (2002a) $\mathrm{Ku}$ affects the ataxia and Rad 3-related/Chk1-dependent S-phase checkpoint response after camptothecin treatment. Cancer Res 62: 2483-2487

Wang J-L, Wang X, Wang H, Iliakis G, Wang Y (2002b) CHK1-regulated S-phase checkpoint response reduces camptothecin cytotoxicity. Cell Cycle 1: 267-272

Wang X, Khadpe J, Hu B, Iliakis G, Wang Y (2003) An over-activated ATR/CHK1 pathway is responsible for the prolonged $G_{2}$ accumulation in irradiated AT cells. J Biol Chem 278: 30869-30874

Xu B, Kim S-T, Lim D-S, Kastan MB (2002) Two molecularly distinct $\mathrm{G}_{2} / \mathrm{M}$ checkpoints are induced by ionizing radiation. Mol Cell Biol 22: $1049-1059$

Zhao H, Watkins JL, Piwnica-Worms H (2002) Disruption of the checkpoint kinase $1 /$ cell division cycle $25 \mathrm{~A}$ pathway abrogates ionizing radiation-induced $\mathrm{S}$ and $\mathrm{G}_{2}$ checkpoints. Proc Natl Acad Sci USA 99: $14795-14800$ 